\title{
EVALUACIÓN DE DIFERENTES METODOLOGÍAS PARA LA CALIBRACIÓN DE LAS ESTIMACIONES DE PRECIPITACIÓN CMORPH SOBRE SUDAMÉRICA
}

\author{
JUAN JOSÉ RUIZ
}

\author{
Centro de Investigaciones del Mar y la Atmósfera (CONICET-UBA), Departamento de Ciencias de la \\ Atmósfera y los Océanos, Universidad de Buenos Aires, Argentina \\ jruiz@cima.fcen.uba.ar
}

Recibido Febrero 2008 - Aceptado Junio 2009

\section{RESUMEN}

\begin{abstract}
Este trabajo describe la aplicación de diferentes técnicas para la calibración de estimaciones de precipitación en base a microondas pasivas utilizando datos pluviométricos. Los experimentos están orientados al desarrollo de una calibración operativa de las estimaciones de precipitación sobre la región del Sudeste de Sudamérica que puede ser fácilmente extendida a otras regiones. Los resultados indican que los algoritmos utilizados permiten reducir eficientemente los errores sistemáticos presentes en las estimaciones satelitales de precipitación mejorando diversos aspectos del desempeño del producto sobre la región de estudio.
\end{abstract}

Palabras clave: estimación de precipitación, cmorph, salljex

\begin{abstract}
EVALUATION OF DIFFERENT METHODOLOGIES FOR PRECIPITATION ESTIMATES CALIBRATION - CMORPH - OVER SOUTH AMERICA This work describes the application of several methods for the calibration of precipitation estimates generated from passive microwave sensors using rain gauge observations. The experiments evaluated strategies for the development of an operational calibration process over Southeastern South America which can be easily applied to other regions. Results show that the proposed calibration algorithm can effectively reduce the systematic errors in the precipitation estimates leading to an improvement on its reliability over the study region.

Keywords: precipitation estimates, CMORPH, SALLJEX
\end{abstract}

\section{INTRODUCCIÓN}

De acuerdo con lo señalado por Ebert et al. (2007), conocer la distribución espacial y temporal de la precipitación es de suma importancia para diversas actividades entre las que se cuentan aquellas relacionadas con el pronóstico del tiempo, la hidrología, la industria y el agro. Durante los últimos años surgieron diversas técnicas para estimar la precipitación acumulada en base a sensores remotos y a una combinación de sensores remotos y pluviómetros (Joyce et al., 2004; Huffman et al., 2005; Huffman et al., 2007; entre otros) que buscan lograr una descripción detallada de la variabilidad temporal y espacial de la precipitación aún en aquellas regiones donde la densidad de estaciones es muy escasa.

Uno de los mayores avances en este campo, fue la incorporación en varios satélites (TRMM, SSM/I, NOAA) de sensores en microondas pasivas que proveen mejores estimaciones de la tasa de precipitación (Kidd et al., 2003). En particular una de las estimaciones que hace uso de esta nueva tecnología es el CMORPH (CPC Morphing Technique) (Joyce et al., 2004). Las estimaciones CMORPH están disponibles en Internet con diferentes resoluciones espaciales y temporales desde diciembre de 2002 (http://www.cpc.ncep.noaa.gov/ products/janowiak/cmorph_description.html).

Dentro de la región de Sudamérica, existen áreas en donde la densidad de estaciones pluviométricas es muy escasa y la medición de la precipitación se hace en el mejor de los casos cada 6 horas. La falta de datos en gran parte de nuestra región, dificulta desarrollos de aplicaciones, la verificación de los pronósticos numéricos (Saulo y Ferreira, 2002; Seluchi y Chou, 2000; Campetella y Saulo, 2003) y la calibración de pronósticos probabilísticos que tienen la potencialidad de mejorar la calidad 
del producto final que se brinda a los usuarios (Ruiz et al., 2009). Por otra parte, un conocimiento detallado de la distribución espacial de la precipitación es fundamental para la estimación de la humedad del suelo en la región, variable que a su vez tiene un gran impacto en la calidad de los pronósticos regionales a corto plazo (Gevaerd y Freitas, 2006). Otra aplicación importante, es la utilización de los datos de precipitación para la estimación de los caudales de los ríos de la región mediante la utilización de modelos hidrológicos (Saurral, 2008). En este punto en particular, Su et al. (2008) mostraron que sobre nuestra región la utilización de estimaciones de precipitación basadas en una combinación de microondas pasivas y datos pluviométricos dio buenos resultados en la simulación de caudales de las distintas sub-cuencas que componen la cuenca del Plata.

Las actividades antes mencionadas, requieren una representación confiable de la precipitación. Para saber si las estimaciones CMORPH pueden ser utilizadas en el marco de estas aplicaciones sobre la región de Sudamérica, es necesario conocer sus errores sistemáticos y desarrollar algoritmos de calibración que permitan reducirlos o eliminarlos. Es por eso que el objetivo planteado en este trabajo es evaluar diferentes estrategias para la calibración de las estimaciones CMORPH que permitan mejorar la confiabilidad de las mismas mediante la reducción de la componente sistemática del error. Para esto es necesario en primer lugar analizar como son los errores sistemáticos que presentan dichas estimaciones en forma análoga a como se ha realizado para otras regiones del planeta (Ebert et al., 2007; Joyce et al., 2004) y para otras estimaciones de precipitación que utilizan microondas pasivas ( $\mathrm{Su}$ et al., 2008). En particular para la región de Sudamérica, estudios preliminares indican que los datos CMORPH tienden a sobreestimar los acumulados de precipitación (Ruiz et al., 2009).

El análisis se centró en la estación cálida 2002-2003 durante la cual se llevó a cabo el experimento de campo SALLJEX (Vera et al., 2006). Este experimento contó con una red pluviométrica compuesta por mediciones obtenidas con la red operativa, información aportada por diferentes instituciones de los países participantes y estaciones instaladas especialmente con el fin de incrementar la cobertura espacial de la red. La mayor densidad de estaciones asociadas a esta red se ubicó sobre la región del Noreste de Argentina, Paraguay y Uruguay. El área seleccionada para el presente trabajo abarca la región antes mencionada y el sur de Brasil, en donde se utilizaron los datos de la Agencia Nacional de Aguas (ANA). Si bien la mayoría de los experimentos se desarrollaron durante este período en el cual había gran disponibilidad de datos, el desempeño de las calibraciones también fue evaluado durante otros períodos en los cuales solo se contaba con datos de la red operativa que presenta una densidad mucho menor. Asimismo, si bien todos los experimentos fueron realizados sobre el Sudeste de América del Sur, las metodologías ensayadas son fácilmente aplicables a diferentes regiones.

\section{DATOS Y METODOLOGÍA:}

\subsection{Datos de la red pluviométrica:}

Los datos de precipitación de la red pluviométrica utilizada en el presente trabajo proveen en su mayoría valores de precipitación acumulada en 24 horas medidos a las 12 UTC. En este caso se utilizó la versión 3 de los datos de precipitación del experimento SALLJEX que fue consistida utilizando imágenes satelitales infrarrojas y datos de precipitación de estaciones cercanas como se describe en Penalba et al. (2004). Durante dicho proceso de consistencia, se identificaron datos potencialmente erróneos los cuales no fueron utilizados en el proceso de verificación/calibración propuesto en este trabajo.

Como se describió previamente, sobre la región del sur de Brazil, se utilizaron los datos de la red ANA. Estos datos fueron consistidos previamente como se describe en Liebmann y Allured (2005).

Los datos provenientes de las redes SALLJEX y ANA fueron llevados a una retícula Lambert con $40 \mathrm{~km}$ de resolución, esta retícula presenta la ventaja de que el área asociada a cada punto de retícula permanece casi constante con la latitud. El método de interpolación utilizado fue el de promedio por cajas que fue utilizada en otros estudios de comparación entre estimaciones satelitales y observaciones de superficie como por ejemplo Chokngamwong y Chiu (2008). Este método consiste en tomar cada punto de la retícula elegida, y definir una región (caja) que coincide con el área representada por dicho punto (las áreas de los puntos de retícula vecinos son adyacentes pero no se superponen). Dentro de cada caja se computa el valor medio diario de la precipitación (considerando todos los datos de los pluviómetros que se encuentran dentro de los límites de la caja) y el número de pluviómetros que informan en cada día en particular. En aquellas cajas en donde no se cuenta con datos pluviométricos el valor de precipitación se considera faltante y no se utiliza en la calibración.

Finalmente para obtener datos más confiables para la verificación y calibración de las estimaciones de precipitación CMORPH sobre la región se fijaron algunos criterios para seleccionar los puntos más confiables y representativos. Consecuentemente, se decidió eliminar las cajas que cumplieran alguna de estas condiciones:

- El número de pluviómetros en la caja era menor que 2.

- El porcentaje de observaciones faltantes en la caja era superior al $75 \%$.

- No hubo ningún reporte de no precipitación en todo el período considerado. 
También se eliminaron las observaciones individuales que indicaran valores de precipitación acumulada superiores a $400 \mathrm{~mm}$ en 24 horas antes de realizar el promedio por cajas (este criterio solo descartó 2 observaciones en todo el período considerado). La aplicación de estas restricciones limitó la disponibilidad de datos fuera de la región centro-norte de Argentina, Uruguay, Paraguay y sudeste de Brasil. De todas maneras, y dados los objetivos del trabajo, se considera que es preferible restringir el dominio de análisis, preservando los requerimientos de cantidad y calidad de observaciones disponibles por unidad de área, que cubrir un área mayor.

El período de datos considerado va del 15 de diciembre de 2002 al 15 de febrero de 2003. Si bien el experimento SALLJEX comenzó el 15 de noviembre de 2002, las estimaciones del CMORPH comenzaron un mes después, por lo que se eligió el 15 de diciembre como fecha inicial para este estudio. La Figura 1 muestra la distribución espacial del número de observaciones disponibles luego de aplicar los criterios descritos más arriba en el período considerado para el presente trabajo.

\subsection{Datos CMORPH}

El algoritmo CMORPH (NOAA CPC Morphing Technique) (Joyce et al., 2004) está basado en el uso de microondas pasivas para estimar la tasa de precipitación asociada a los sistemas nubosos y al uso de las imágenes infrarrojas de los satélites GOES para estimar el desplazamiento de los mismos. La estimación basada en microondas pasivas resulta ser más exacta que otras estimaciones basadas exclusivamente en la temperatura de los topes nubosos (Joyce et al., 2004; Ebert, 2007), esto se debe a que por ejemplo en los grandes sistemas precipitantes, los topes fríos pueden extenderse a un área mucho mayor que la región en donde efectivamente están ocurriendo precipitaciones, por otra parte también pueden registrarse precipitaciones muy intensas que provengan de nubes con un desarrollo vertical menor y que por ende tengan una temperatura en el tope más alta. Por otro parte, la radiación emitida en diferentes bandas de las microondas permite estimar el contenido de agua líquida y de hielo en las nubes, lo cual presenta una relación mucho más directa con las tasas de precipitación en superficie. Como desventaja principal se puede mencionar que hasta el momento los sensores en microondas pasivas no se encuentran a bordo de los satélites geoestacionarios, esto hace que no se tenga una cobertura temporal tan densa como la que se puede obtener en este tipo de plataformas. En el caso del algoritmo CMORPH este aspecto está parcialmente corregido ya que se utiliza la información proveniente de los satélites geoestacionarios para realizar un seguimiento de los sistemas precipitantes y poder conocer su posición con mayor resolución temporal. Estas estimaciones cuentan con una resolución espacial máxima de 0.072 grados y una resolución temporal máxima de media hora. Para el presente estudio, se partió de los productos con una resolución intermedia de 0.25 grados y acumulados en

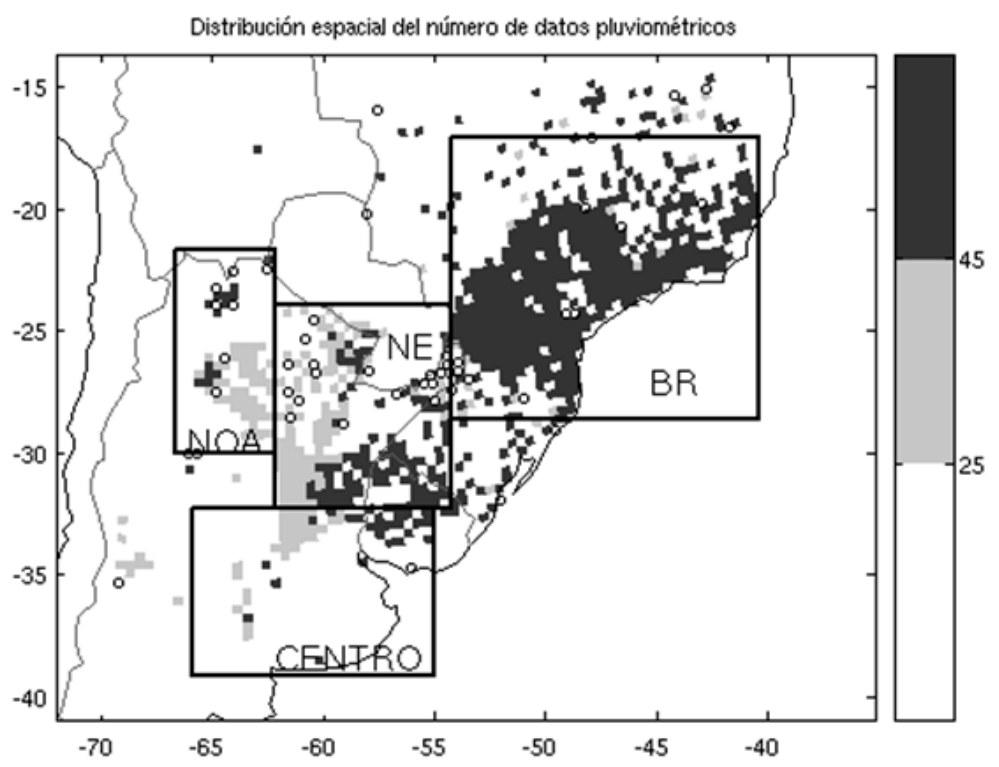

Figura 1 - Número de observaciones disponibles en las cajas que cumplen con los criterios establecidos para la red de pluviómetros. Los rectángulos negros indican la posición de las diferentes regiones discutidas en el texto. Los círculos blancos indican la posición de las cajas en donde el número de datos es inferior a 25 . 
períodos de 3 horas, que luego fueron acumulados sobre 24 horas y promediados sobre las mismas cajas definidas para los datos pluviométricos utilizando la misma metodología del promedio por cajas. Si bien la utilización del promedio por cajas no está tan justificada en este caso debido a que la resolución de la retícula nativa del CMORPH y la retícula utilizada en este trabajo son similares, los resultados obtenidos no se modificaron significativamente en el caso en el que se utilizó una técnica de interpolación lineal.

\subsection{Parámetros seleccionados para la verificación}

Como primer paso para estudiar la relación entre las observaciones de precipitación y las estimaciones del CMORPH, se verificó la calidad del CMORPH contra las observaciones en base al cálculo de diferentes parámetros que permitieran analizar las diferencias entre ambos conjuntos de datos.

Para comenzar a aislar la componente sistemática de las diferencias entre el CMORPH y los datos pluviométricos, se calcularon por un lado medias de precipitación en milímetros por día sobre todo el período considerado para ambos conjuntos de datos y se realizó un estudio de la evolución temporal de la precipitación media areal por regiones. También se estudió la distribución espacial de las frecuencias de precipitación.

Para obtener medidas de la bondad del ajuste entre ambos conjuntos de datos, se calcularon estadísticos como el error cuadrático medio (ECM) y el coeficiente de correlación lineal (R). Se emplearon, además, medidas frecuentemente utilizadas en la evaluación de la calidad de los pronósticos numéricos de precipitación como el Equitable Treath Score (ETS) (Schaefer, 1990) (Ecuaciones 1 y 2). Para el cálculo de este índice el rango de valores precipitación acumulada se separa en umbrales y para cada umbral se calculan las cantidades de acierto, sorpresa y falsa alarma, donde acierto representa la cantidad de casos en los que tanto la precipitación estimada como la observada estuvieron por encima del umbral seleccionado, sorpresa indica la cantidad de casos en los que la lluvia observada estuvo por encima del umbral, pero la lluvia estimada estuvo por debajo, falsa alarma indica la cantidad de casos en los que la estimación estuvo por encima del umbral, pero la lluvia observada estuvo por debajo y total el número de pares estimación-observación considerados. El índice ETS tiene un valor máximo de 1 que corresponde a una estimación perfecta. Un valor de 0 en el ETS representa una estimación en donde el número de aciertos es similar al que se obtendría por azar expresado por la cantidad acierto $_{\mathrm{r}}$. Por otra parte también se calculó el sesgo o el error en frecuencia, como el cociente entre la frecuencia estimada de un evento y la frecuencia observada (Ecuación 3), valores de sesgo cercanos a 1 indican que la frecuencia de ocurrencia de lluvias por encima de un determinado umbral en la estimación es similar a lo observado, valores inferiores indican un déficit mientras que valores mayores que uno indican una sobreestimación de la frecuencia de dichos eventos. El sesgo no aporta información sobre la coincidencia entre las áreas de precipitación estimada y observada esto por un lado representa una desventaja ya que no permite evaluar la precisión de las estimaciones con respecto a la posición de los sistemas precipitantes, no obstante debido a esta característica presenta la capacidad de detectar errores sistemáticos en la estimación independientemente de los errores de posición que puedan estar presentes. Por otra parte el ETS provee una medida que es sensible a los errores de posición y a los errores en frecuencia. Estas medidas han sido utilizadas extensamente en la literatura y en particular Hossain y Huffman (2008) estudiaron su aplicación a la estimación de los errores asociados con las estimaciones de precipitación derivadas de sensores remotos concluyendo que las mismas eran adecuadas para ser utilizadas en las escalas espaciales que se utilizan en este trabajo.

$$
\begin{aligned}
& E T S=\frac{\text { acierto }- \text { acierto } r}{\text { acierto }+ \text { sorpres } a+\text { falsa alarma }- \text { acierto }_{r}} \\
& \text { acterto }_{r}=\frac{(\text { acierto }+ \text { sorpresa })(\text { acierto }+ \text { falsa alarma })}{\text { total }} \\
& \text { sesgo }=\frac{\text { acierto }+ \text { sorpres } a}{\text { falsa alarma }+ \text { acierto }}
\end{aligned}
$$

Tanto el ETS como el sesgo, fueron calculados conformando un gran conjunto de datos a partir de todas las observaciones/estimaciones diarias en cada uno de los puntos de retícula. El resultado obtenido es un parámetro global integrado tanto espacial como temporalmente.

\subsection{Estrategias de calibración:}

El objetivo del presente estudio es la obtención de un algoritmo de calibración que permita remover gran parte de la componente sistemática del error en los datos CMORPH como función de la intensidad de precipitación estimada. Las características de dicho error sistemático se muestran en la sección de resultados. Para lograr este objetivo se plantearon a priori diferentes estrategias, las cuales fueron evaluadas utilizando los mismos parámetros que sirvieron para la verificación de los CMORPH contra los datos pluviométricos.

Se llevaron a cabo 4 estrategias de calibración diferentes las cuales se detallan a continuación:

- Calibración por valores medios (CAL I): Se separa el 
rango de la variable precipitación estimada en categorías. Cada vez que la estimación CMORPH cae dentro de una determinada categoría, se toma el valor observado correspondiente y se lo agrega al conjunto de observaciones vinculadas a dicha categoría. De esta forma para cada intervalo o categoría de precipitación estimada se tiene un conjunto de observaciones asociado (conjunto de verificación). Luego, para cada categoría se computa la media del conjunto de verificación y ese valor es el que se asigna como nuevo valor estimado o corregido al valor central de cada categoría. Para corregir un valor estimado por el CMORPH, se interpola linealmente el valor de las correcciones obtenidas al valor exacto estimado por el CMORPH y se reemplaza la estimación por el valor corregido.

- Calibración por azar (CAL II): Se separa la variable estimada en categorías y se obtiene el conjunto de verificación como en el caso anterior, solo que en lugar de corregir usando el valor medio del conjunto de verificación de cada categoría, se reemplaza el valor estimado por un elemento del conjunto de verificación seleccionado al azar. Es importante destacar que dicho valor se selecciona para cada punto al momento de hacer la calibración usando un generador de números aleatorios uniformemente distribuido. Este procedimiento se basa en el algoritmo desarrollado por Stensrud y Yussouf (2007) para la calibración de pronósticos probabilísticos.

- Regresión lineal (CAL III): Este método se basa en la obtención de una recta de regresión entre ambos conjuntos de datos utilizando el método cuadrados mínimos. Previo al cálculo de la regresión, se definió una retícula con $2.5^{\circ} \mathrm{x}$ $2.5^{\circ}$ de resolución horizontal y se calculó la precipitación media areal diaria estimada y observada en cada una de esas cajas mediante el método de promedio por cajas. Estas cajas se situaron en la región donde la densidad de estaciones era mayor. Se retuvieron solo los valores de precipitación media provenientes de cajas con más de 30 datos. Sobre los valores medios areales correspondientes a las cajas de $2.5^{\circ} \times 2.5^{\circ}$ de ambos conjuntos de datos, se computaron los coeficientes de la recta que mejor relaciona ambos conjuntos y se utilizó esa función como calibración con la salvedad de que al momento de calcular los valores corregidos se anula la ordenada al origen (que de todas formas es un valor del orden de $1 \mathrm{~mm}$ ) para salvar la inconsistencia que esto genera para valores de precipitación estimada cercanos a $0 \mathrm{~mm}$.

- Método de eliminación del sesgo (CAL IV): Este método, está basado en el trabajo de Hamill (1999) y es un algoritmo que remueve el sesgo de un conjunto de datos respecto de otro. Para diferentes valores de precipitación (umbrales) se busca el valor de precipitación estimada tal que el sesgo con respecto a dicho umbral observado sea 1 . Para encontrar dicho valor, se deja fijo el valor umbral utilizado como verificación (por ejemplo precipitaciones observadas mayores a $2.5 \mathrm{~mm}$ ), y luego comienzan a utilizarse diversos valores de precipitación estimada como estimadores de la lluvia por encima de 2.5 $\mathrm{mm}$ (por ejemplo se calcula el sesgo que resulta de estimar las
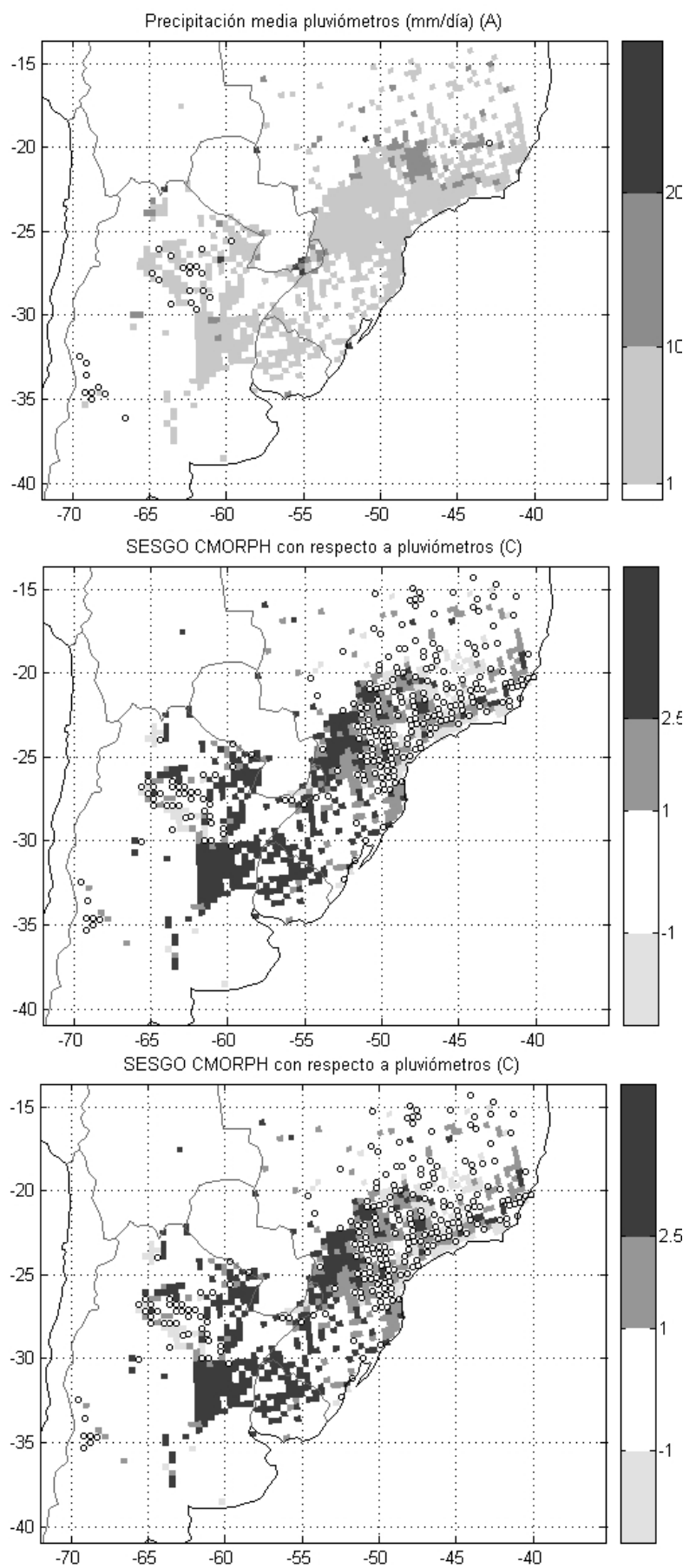

Figura 2 - Precipitación media sobre el período considerado para (a) datos pluviométricos, (b) estimaciones CMORPH y (c) diferencia entre ambos (CMORPH-PLUVIÓMETROS). Los valores medios se calcularon sobre los datos que verificaron los criterios descritos en la sección datos y metodología. Los círculos blancos indican las estaciones cuyos valores corresponden al color blanco de la escala. 
precipitaciones mayores a $2.5 \mathrm{~mm}$ utilizando precipitaciones estimadas mayores a $5 \mathrm{~mm}$ ). Para cada valor de precipitación estimada utilizado, se obtiene un valor de sesgo diferente, hasta encontrar uno que esté cercano a 1 . En el proceso de calibración se asigna el valor del umbral observado al valor de estimación tal que el sesgo resulta ser uno para dicho valor observado. Es decir que si el valor estimado de $5 \mathrm{~mm}$ resultó tener un sesgo de 1 con respecto al umbral observado de $2.5 \mathrm{~mm}$, cada vez que el valor de la estimación sea $5 \mathrm{~mm}$ se reemplazará este valor por el de $2.5 \mathrm{~mm}$.
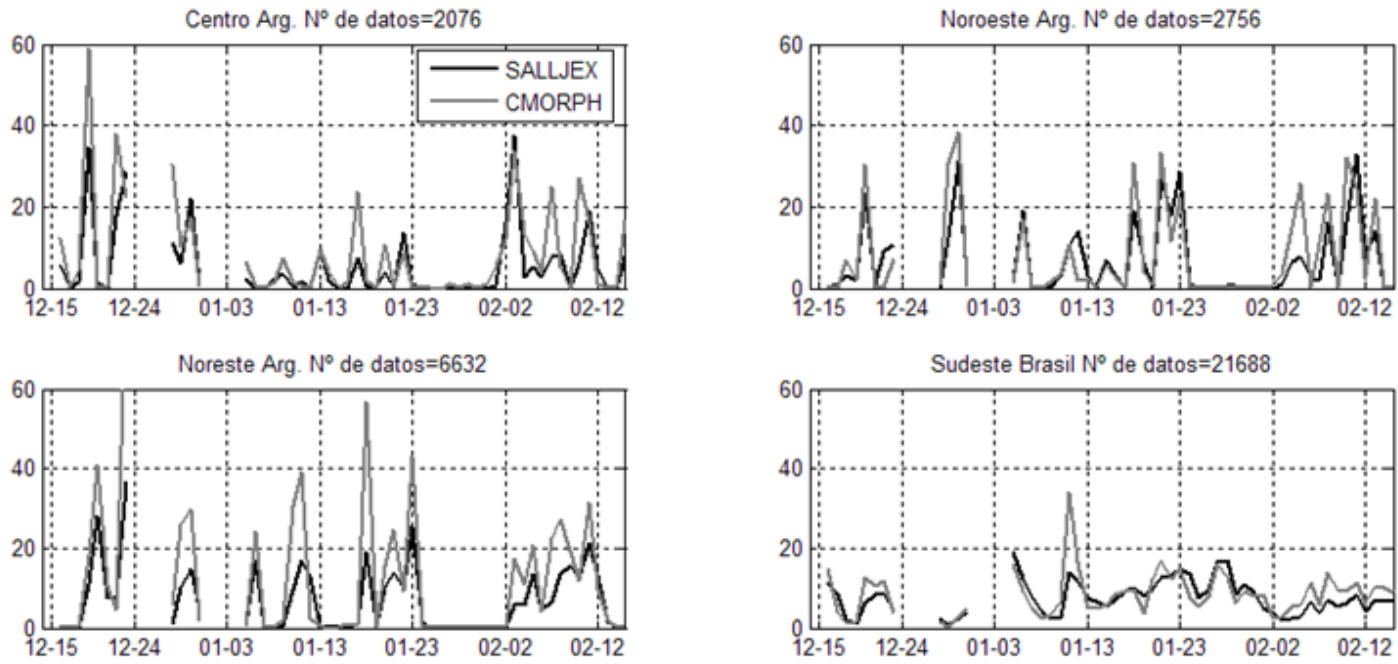

Figura 3 - Evolución temporal de la precipitación media areal sobre diferentes regiones de Sudamérica en base a los datos pluviométricos (línea negra) y en base a las estimaciones CMORPH (línea gris). (a) Región centro de Argentina, (b) región Noroeste de Argentina, (c) Región Noreste de Argentina, (d) Región Sudeste de Brasil. Los números en la parte superior de los gráficos indican el número de datos totales en cada región en el período considerado.

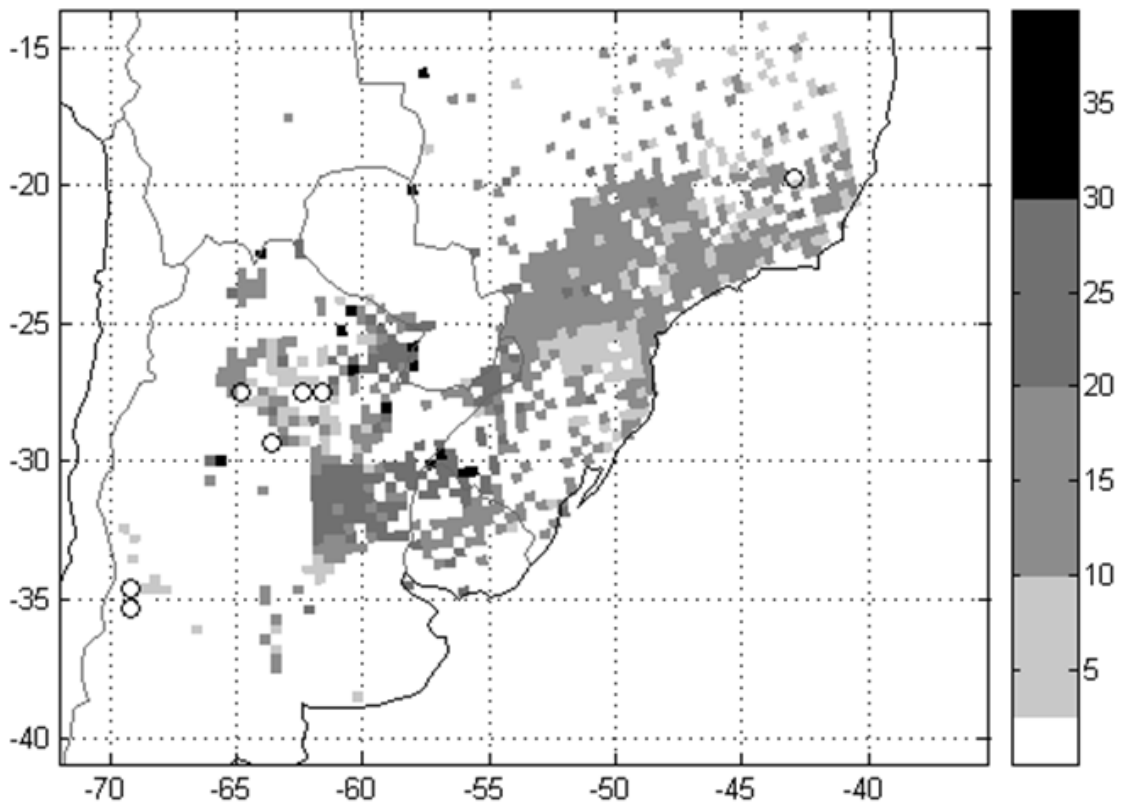

Figura 4 - Distribución espacial del ECM para las estimaciones CMORPH no calibradas sobre todo el período considerado. Los círculos blancos indican la posición de las estaciones en donde el valor del ECM es inferior a $2.5 \mathrm{~mm}$. 
Para una primera evaluación de las calibraciones, se entrenó y se verificó cada calibración con el mismo conjunto de datos. Luego se tomó la calibración que produjo el mejor resultado y se realizó un nuevo experimento en donde el primer mes del período considerado fue utilizado para la calibración de los datos y el segundo fue utilizado para verificar los resultados de dicha calibración y evaluar si los resultados de la calibración son aplicables a otros períodos al menos dentro de la misma estación. También se realizaron experimentos para determinar el desempeño del algoritmo en el caso de contar con una red significativamente menos densa que la empleada en este estudio, como es la red operativa sobre la región (en particular sobre el centro y norte de Argentina donde la densidad de datos es menor).

\section{RESULTADOS}

\subsection{Verificación de las estimaciones CMORPH:}

En primer lugar se analizó el comportamiento medio de ambos conjuntos de datos. La Figura 2, muestra la distribución espacial de la precipitación media en cada punto de retícula para los datos pluviométricos, el CMORPH y la diferencia entre ambos. Es importante destacar que dada la gran inhomogeneidad en la distribución de los datos pluviométricos (sobre todo en el centro y norte de Argentina) (Ver Figura 1), esta figura no es representativa de la distribución espacial de la precipitación media sobre el período, pero sí permite analizar las diferencias entre ambos conjuntos de datos. Como se puede apreciar en la Figura 2c, el CMORPH tiende a sobreestimar la precipitación media sobre el este de Argentina, Uruguay y en Brasil cerca de la frontera con Paraguay. Como una primera aproximación al problema, todos los datos de la región de estudio se utilizan para el proceso de calibración y verificación, de forma tal que si existe alguna dependencia regional en los errores sistemáticos esta no está siendo tenida en cuenta en los algoritmos propuestos, aunque podría incluirse fácilmente si hubiese suficiente información pluviométrica. En dicho caso se podría dividir el dominio en regiones que cumplan con la condición de que el comportamiento de los errores sistemáticos de la estimación dentro de cada región sea lo más homogénea posible, luego se desarrolla una calibración para cada región utilizando solamente las estimaciones y observaciones provenientes de dicha región.

Para comprobar si el CMORPH captura adecuadamente los eventos de precipitación y sus intensidades relativas, se analizó la evolución de la precipitación media areal en diferentes regiones cuya posición está indicada en la Figura 1. La selección de las regiones se basa en la existencia de regímenes de precipitación diferentes. Los resultados se muestran en la

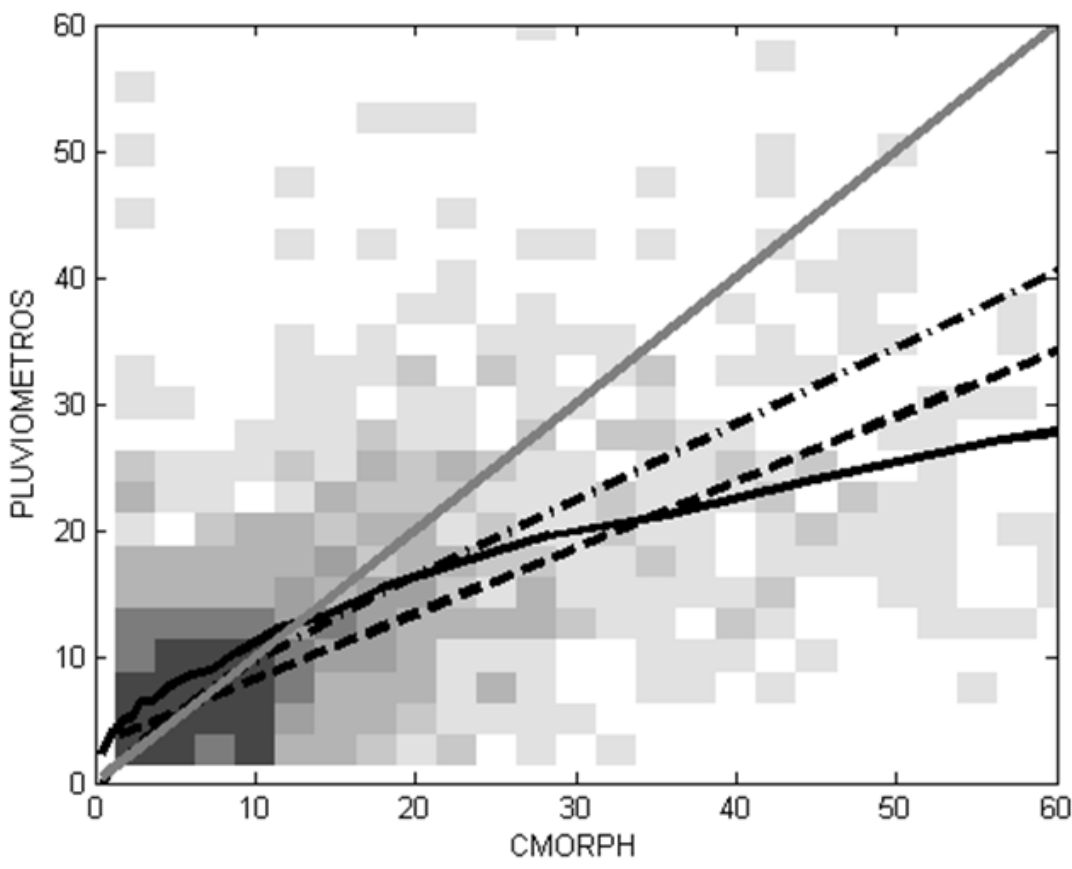

Figura 5 - Densidad de puntos en un diagrama de dispersión PLUVIOMETROS vs CMORPH para las cajas de $2.5^{\circ}$ x $2.5^{\circ}$ (Ver discusión en el texto). Líneas: relación PLUVIÓMETROS-CMORPH según las diferentes calibraciones utilizadas, valores medios (CAL I, línea continua negra), correlación lineal (CAL III, línea a trazos), y reducción del sesgo (CAL IV, línea trazo-punto). La línea continua gris indica la relación 1 a 1. 
Figura 3. Como se puede ver en la figura, el CMORPH captura adecuadamente los eventos de precipitación en cada una de las regiones analizadas como así también existe una buena relación en la intensidad relativa de cada uno de los eventos. No obstante y en concordancia con lo observado en la Figura 2, se observa una sobreestimación de los valores acumulados para la mayoría de los eventos. Esta sobreestimación es más evidente cuando se considera la porción noreste del dominio.

La Figura 4, muestra la distribución espacial del ECM. En este caso se puede observar que los valores de ECM son superiores a la componente sistemática del error. Esto pone ciertos límites a las mejoras que podrían lograrse con una calibración que solo remueva la componente sistemática del error, dado que todavía existirían errores grandes para la estimación puntual de los acumulados de precipitación.

La Figura 5 muestra un diagrama de dispersión de los datos pluviométricos en función de los datos CMORPH para la precipitación media en cajas de $2.5^{\circ} \times 2.5^{\circ}$ correspondientes a la CAL III. Este diagrama de dispersión se muestra como la densidad de puntos en intervalos de $2.5 \times 2.5 \mathrm{~mm}$ de forma tal que la interpretación del diagrama sea más clara. Se puede apreciar que existe una importante dispersión en la estimación de la precipitación acumulada diaria por parte de los CMORPH. Esto corrobora que si bien una corrección basada pura y exclusivamente en el valor de la estimación, puede mejorar el producto, todavía queda una importante porción de la variabilidad de los errores que no puede ser explicada. Este tipo de comportamiento es esperable, dado que es sabido que la precipitación tiene una importante variabilidad espacio-temporal, que dificulta su estimación y eventualmente su pronóstico. La dispersión asociada a los promedios areales sobre las cajas de $40 \mathrm{~km}$ de resolución es aún mayor (no se muestra).

Por otra parte, en la Figura 5 se incluyen las diferentes curvas de calibración obtenidas mediante las distintas metodologías ensayadas. La única calibración para la cual no se puede construir una curva de este tipo es la CAL II, ya que para cada caso se utiliza un valor diferente mediante la selección de un elemento al azar. Si analizamos la curva correspondiente a la calibración por valores medios (CAL I) se observa que la precipitación observada para diferentes rangos del CMORPH es mayor que la estimada cuando la misma es pequeña (menor a 10 $\mathrm{mm}$ ) y es muy inferior cuando la lluvia estimada es alta (mayor a $10 \mathrm{~mm}$ ), inicialmente esto induce a pensar que el CMORPH sobreestima los valores altos de precipitación y subestima los valores bajos, no obstante un patrón muy similar puede obtenerse como resultados de errores en el posicionamiento de los sistemas precipitantes (no se muestra). Estos errores en el caso de los CMORPH podrían surgir por el hecho de que no siempre se cuenta con estimaciones de microondas para determinar la posición de los sistemas y que cuando no se cuenta con dichos productos la posición es estimada a partir de las imágenes infrarrojas. Un efecto similar se observa en la CAL III. La CAL IV por otra parte se basa en la corrección del sesgo
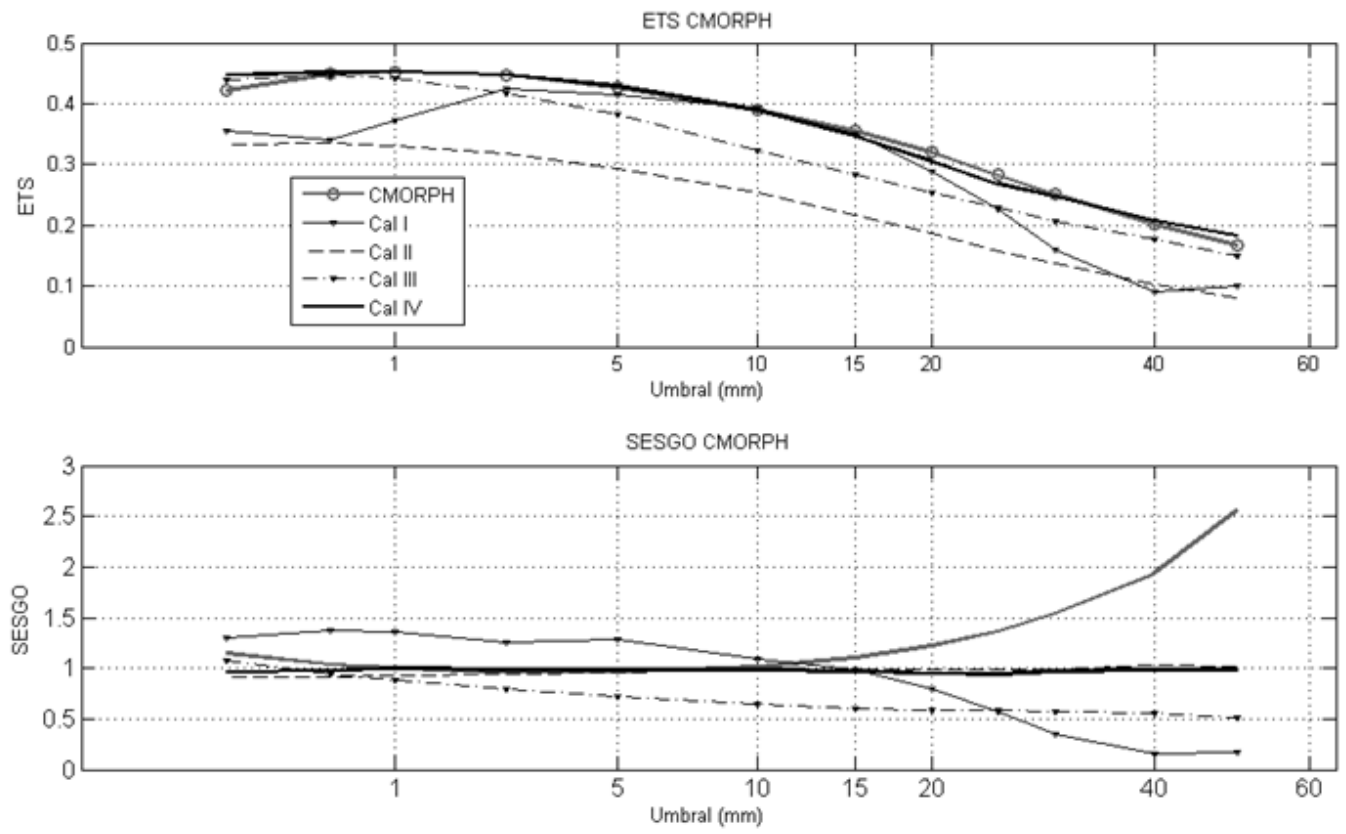

Figura 6 - Gráfico de ETS y sesgo para el CMORPH y las diferentes calibraciones ensayadas en base a los datos pluviométricos sobre todo el período considerado. La escala del eje X ha sido transformada para permitir una mejor visualización de lo que sucede en los umbrales de precipitación más bajos. 
y no es sensible a los errores de posición. Se puede apreciar que la curva asociada a esta calibración tiene una pendiente mayor debido a que no se ve afectada por los errores de posición sino solamente por las diferencias en las frecuencias asociadas a los diferentes valores de precipitación acumuladas. El estudio del impacto que diferentes fuentes de error (posición, intensidad, etc.) tienen sobre las medidas de error utilizadas en un marco teórico y su aplicación a la interpretación de los patrones de error obtenido para situaciones reales como las analizadas en este estudio es parte de un trabajo en preparación.

Para ver el impacto que la calibración tuvo sobre la calidad de las estimaciones de precipitación, se calculó el ETS y el sesgo sobre todo el período para las estimaciones del CMORPH sin calibrar y para las diferentes calibraciones. Para la verificación se utilizó, como primera aproximación, el mismo conjunto de datos utilizado para la calibración. Más adelante se mostrarán resultados en donde el conjunto de datos utilizado para la calibración de la estimación es diferente al utilizado para la verificación.
La Figura 6 muestra los valores de ETS y sesgo en función del umbral de precipitación considerado para las estimaciones CMORPH y para las diferentes calibraciones consideradas Los valores de ETS encontrados, son similares a los obtenidos en estudios previos como Ebert et al. (2007) para la época estival en otras regiones del mundo. Las diferencias, aunque pequeñas, podrían explicarse por la mayor resolución de la retícula utilizada por estos autores (20 km de resolución) (Hossain y Huffman, 2008) y por el método de interpolación utilizado (vecino más cercano). Con respecto a los valores de sesgo, las estimaciones no calibradas muestran un sesgo que es relativamente pequeño para valores bajos de precipitación, pero que crece notablemente para valores de precipitación altos (superiores a $10 \mathrm{~mm}$ ) llegando a un valor de 2,5 para valores de $50 \mathrm{~mm}$ lo que implica casi la triplicación de la frecuencia de ocurrencia de estos eventos en las estimaciones del CMORPH con respecto a las estimaciones pluviométricas. Este comportamiento también fue encontrado para las estimaciones de precipitación TRMM (Huffman et al., 2007) cuando fueron

Tabla 1 - Valores de ECM y coeficiente de correlación (R) para la estimación CMORPH sin calibrar y las diferentes calibraciones.

\begin{tabular}{|l|c|c|}
\hline \multicolumn{1}{|c|}{ Calibración } & ECM & $\mathrm{R}$ \\
\hline No calibrado & 14.9 & 0.63 \\
\hline CAL I & 9.6 & 0.67 \\
\hline CAL II & 13.4 & 0.46 \\
\hline CAL III & 10.3 & 0.63 \\
\hline CAL IV & 10.7 & 0.65 \\
\hline
\end{tabular}
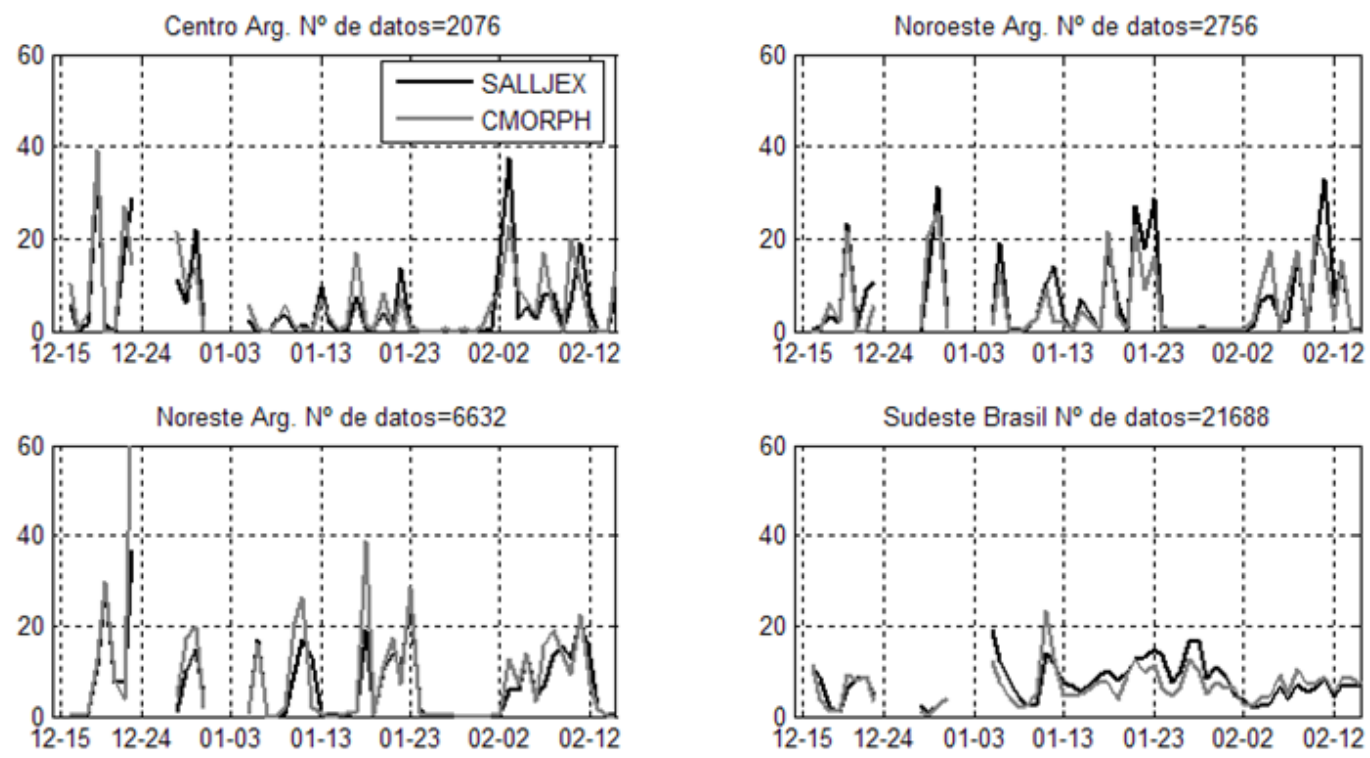

Figura 7 - Como en la Figura 3, pero para la calibración IV. 
analizadas por Su et al. (2007) sobre la región de Sudamérica. La Figura 6, también muestra una sobreestimación en la frecuencia de valores de precipitación por debajo de 1 milímetro.

Con respecto al impacto de las calibraciones sobre el ETS y el sesgo, la Figura 6, muestra que las CAL I y III, tienen un ETS inferior al de la estimación original, lo cual implica una degradación de la calidad de las estimaciones, y valores de sesgo que indican una tendencia a la subestimación en la frecuencia de eventos de precipitación por encima de los 15 $\mathrm{mm}$. Esto se debe básicamente a que la correlación lineal (CAL III) y la relación en la que se basa la CAL I, están afectados por errores como el de posicionamiento que no pueden ser corregidos con una calibración que modifica individualmente cada punto de retícula.

La CAL II, de acuerdo con la Figura 6, muestra un sesgo cercano a 1 para los diferentes umbrales considerados, no obstante, esta calibración muestra un marcado deterioro en el valor del ETS. Este tipo de calibración es la única que introduce la posibilidad de alterar la distribución espacial de la lluvia, ya que las restantes solo modifican la intensidad de la estimación a través de una función de calibración, pero no pueden modificar la forma de los patrones espaciales de la precipitación estimada.

Finalmente la CAL IV muestra, como era de esperarse, un sesgo muy cercano a 1 en todos los umbrales considerados y valores de ETS cercanos a los de la estimación no calibrada.

Otra forma de evaluar el impacto de las diferentes calibraciones, fue calcular el error cuadrático medio (ECM) y la correlación lineal entre las observaciones y las estimaciones. La Tabla 1, muestra dichos valores para las diferentes calibraciones. La mayoría de las mismas produce una reducción del ECM, aunque la menor reducción se obtiene con la CAL II. En cuanto a los coeficientes de correlación, las diferentes calibraciones producen pocos cambios en el mismo a excepción de la CAL II que muestra un deterioro significativo. Estos resultados, junto con los discutidos en la Figura 6, sugieren que la CAL IV es la más apropiada para este caso; de hecho, la reducción del sesgo sistemático es una característica muy deseable para las diferentes aplicaciones discutidas en la introducción de este trabajo. La ventaja de la CAL IV por sobre las demás probablemente se deba a que no responde a errores de posición, pero si responde a errores en la intensidad de las estimaciones, por tanto es más apropiada para el diseño de una calibración que se aplica en cada punto de retícula en forma independiente de lo que sucede en los demás.

La Figura 7 muestra el resultado de aplicar la CAL IV a los cálculos de precipitación media areal sobre las diferentes regiones que se mostraron en la Figura 3. En esta figura se puede apreciar como se reducen los errores en la estimación de precipitación media areal para estas subregiones. No obstante, en la región del NOA se observa una tendencia a la subestimación luego de aplicar la calibración que no estaba presente previamente. Esta tendencia no se observa en las demás subregiones. Este comportamiento sugiere que para obtener mejores resultados, las variaciones espaciales de los errores deberían incorporarse dentro de los algoritmos de calibración. Esto se puede lograr diseñando calibraciones específicas para regiones que comparten un mismo régimen de precipitación o un mismo comportamiento del error sistemático como ha sido discutido previamente.

Resulta de interés evaluar el impacto de CAL IV mediante el cálculo de la distribución espacial del sesgo y del ECM. Los resultados se muestran en la Figura 8. En la Figura 8a, se puede ver que el método de remoción del sesgo como era de esperarse produce mejores resultados en la estimación de la precipitación media reduciendo los errores sistemáticos de los CMORPH que se apreciaban en las estimaciones no calibradas (ver Figura 2c). No obstante, el algoritmo propuesto no logra
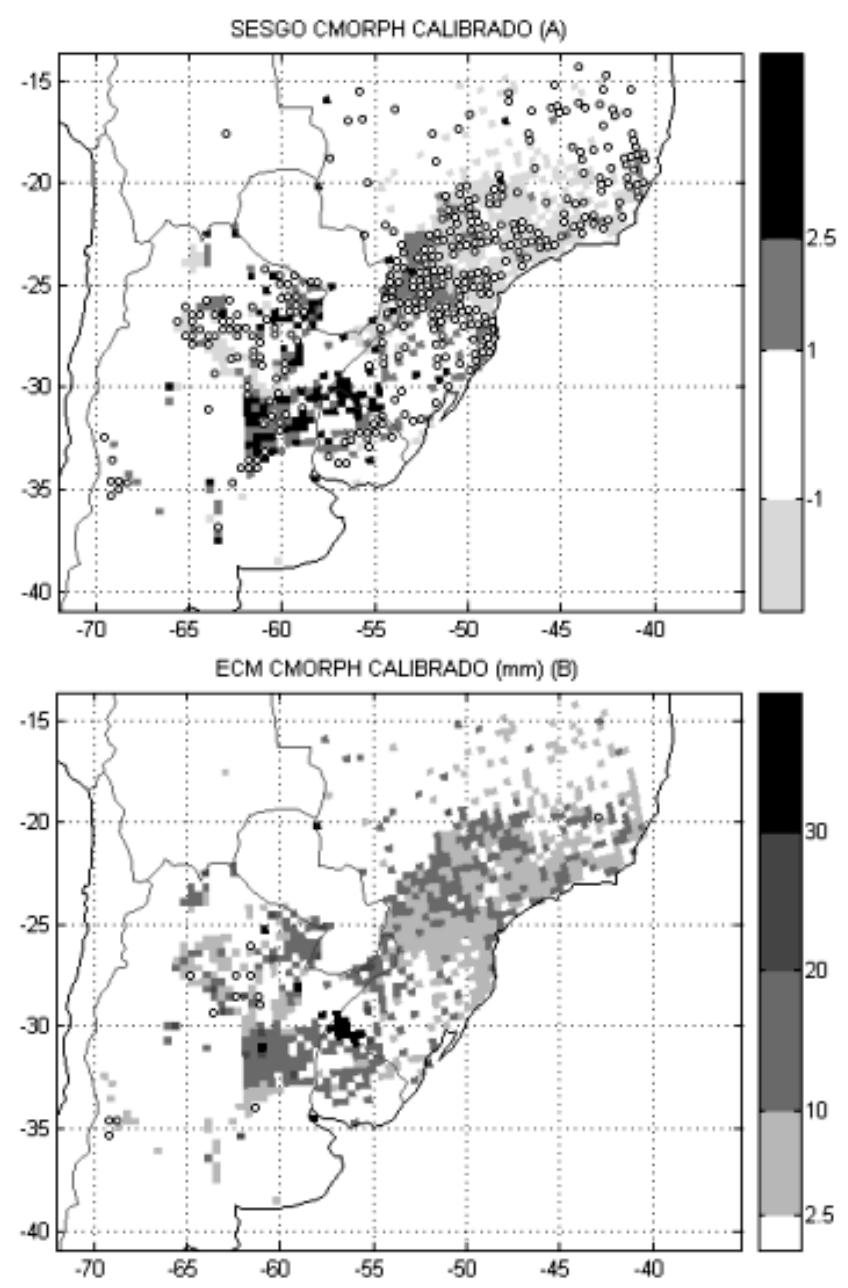

Figura 8 - (a) sesgo en la precipitación media (mm/día) (CMORPHPLUVIÓMETROS) y (b) ECM del CMORPH respecto de los datos pluviométricos para el CMORPH calibrado utilizando CAL IV. 
eliminar totalmente el sesgo húmedo que se observa sobre el este de Argentina y Uruguay e introduce un sesgo más negativo sobre el centro de Brasil que no estaba presente previamente. Este aspecto refuerza la idea de que la calibración debería aplicarse en forma regional. Esta calibración podría introducir por ejemplo una variable extra que sería error sistemático sobre todo el período de entrenamiento de la estación. De esta forma las regiones donde la precipitación es sobreestimada utilizarán una curva de calibración diferente a la de regiones en donde la precipitación media es subestimada. No obstante gran parte del centro y norte de Argentina se comporta de manera bastante homogénea.

La Figura 8b muestra una reducción de los valores de ECM comparados con la estimación no calibrada(verFigura4) que estaría vinculada con la reducción de la componente sistemática del error.

\subsection{Experimentos de sensibilidad a la estación del año y a los datos utilizados en el proceso de calibración}

Los resultados antes mostrados fueron obtenidos para un período particular en el cual la disponibilidad de datos pluviométricos consistidos era mayor que lo habitual sobre el centro y norte de Argentina. Por otra parte, calibración y verificación se realizaron durante el mismo período (es decir con los mismos datos). A continuación se describen una serie de experimentos orientados a evaluar la factibilidad operativa del algoritmo propuesto.
El primer experimento está diseñado para determinar si la calibración podría realizarse con datos de un período correspondiente a la misma estación pero que no coincidiera exactamente con el período de verificación. Para esto se utilizó el primer mes del período considerado ( 15 de diciembre al 15 de enero) como período de calibración o entrenamiento y el segundo ( 15 de enero al 15 de febrero) como período de verificación; este procedimiento se aplicó únicamente a la CAL IV, que mostró ser la más adecuada. La Figura 9 muestra los valores de ETS y sesgo obtenidos de esta forma. Se puede observar, que los valores de sesgo si bien no son exactamente iguales a 1 como en el caso anterior, son bastante cercanos a dicho valor y que el valor del ETS calculado para la estimación calibrada continúa siendo similar a los de la estimación sin calibrar. Este resultado muestra que el método propuesto permite obtener una calibración que puede ser aplicada para períodos dentro de la misma estación fuera del período utilizado para calibrar. Una aplicación directa de este resultado es el desarrollo de una calibración de tipo dinámica en donde la estimación para un día determinado se calibra con los datos de precipitación obtenidos durante los días previos permitiendo el desarrollo de una estimación calibrada en tiempo real. Este procedimiento se está llevando a cabo experimentalmente en el Centro de Investigaciones del Mar y la Atmósfera (CONICET-Universidad de Buenos Aires).

Por otra parte se realizó un experimento en donde los datos utilizados fueron únicamente los de la red GTS (Global Telecomunication System) que constituyen la red operativa de
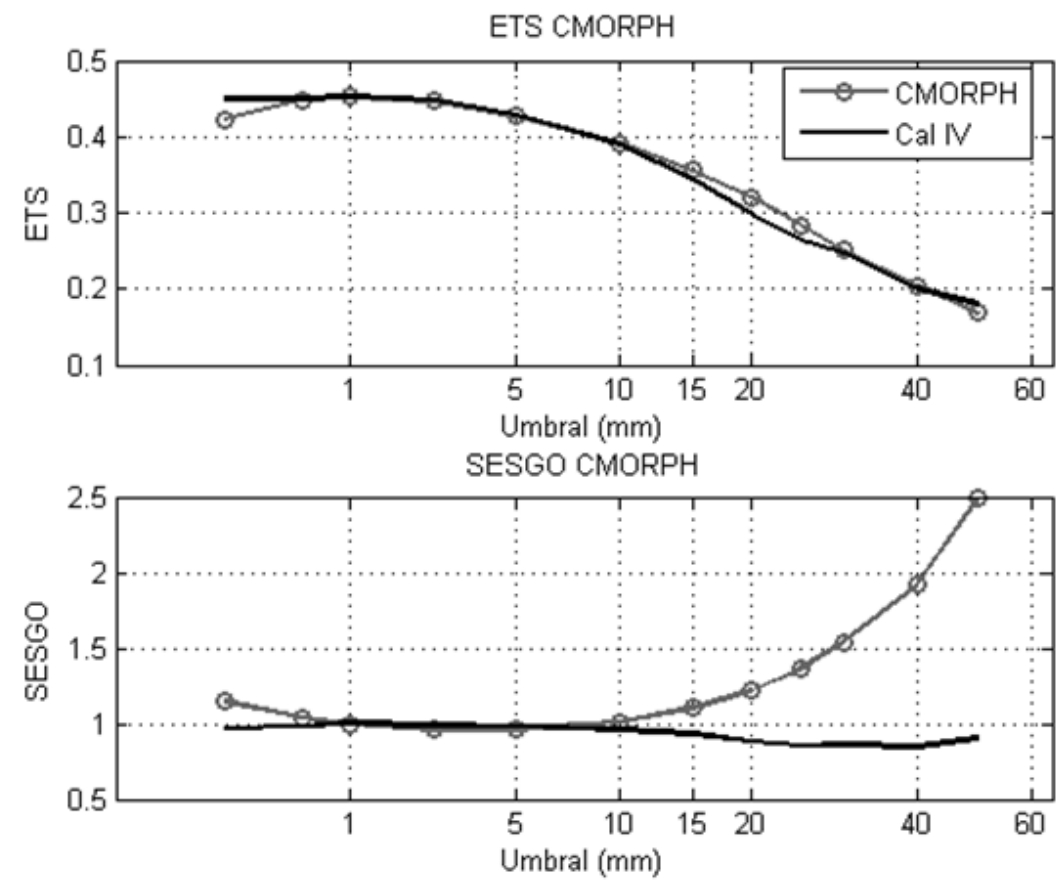

Figura 9 - Como en la Figura 3, pero para la calibración IV. 
medición de precipitación. En el caso de una implementación operativa, la disponibilidad de datos en la región puede ser menor que la utilizada en los experimentos previos; es por eso que es necesario evaluar el impacto de contar con una menor cantidad de observaciones a la hora de calibrar las estimaciones CMORPH. Para esto se utilizaron los mismos períodos de entrenamiento/calibración y de verificación del experimento anterior, pero solo se tuvieron en cuenta los datos de la red GTS. Para poder utilizar estos datos fue necesario relajar los criterios de calibración que exigían una cantidad de estaciones mayor o igual a 2 por cada caja. En este caso la mayoría de las cajas tienen un único dato dado que la red operativa tiene poca densidad sobre la región utilizada en este estudio. Para la verificación de los resultados se utilizó la red SALLJEX para que los mismos fueran comparables con los obtenidos en los experimentos anteriores.

La Figura 10 muestra los resultados obtenidos en términos del ETS y el sesgo para el experimento utilizando los datos del GTS. Como se puede ver en este caso, la calibración con una red de datos menos densa conduce a una subestimación de las frecuencias de lluvias, sobre todo para los umbrales entre 1 y $40 \mathrm{~mm}$. Esta sub-estimación produce un deterioro en los valores de ETS obtenidos. El utilizar una red con menor densidad espacial, no produjo resultados tan satisfactorios como los observados hasta ahora. Para analizar un poco más a fondo las causas de este fenómeno, se analizaron las distribuciones de frecuencias de los diferentes conjuntos de datos.
La Figura 11a, muestra la frecuencia de ocurrencia de diferentes rangos de precipitación observada en pluviómetros para la red GTS, la red SALLJEX + ANA y la red SALLJEX + ANA en los puntos de la red GTS. Comparando los 2 últimos, se observa que hay un comportamiento diferente entre las 2 redes que obedece a las diferencias en la ubicación de las estaciones de ambas. Este cambio también es capturado por las estimaciones CMORPH (no se muestra). No obstante, cuando se comparan las frecuencias de lluvia para los diferentes rangos, para los datos GTS y los datos SALLJEX+ANA en la posición de los datos GTS, se observa que los datos GTS tienden a subestimar las frecuencias de lluvia. La razón de esto no es clara, aunque podría ser que la mayor disponibilidad de pluviómetros en los datos SALLJEX+ANA sobre estas mismas localidades permita detectar eventos de precipitación que no fueron detectados por la red GTS. Estas diferencias podrían ser el origen de los sesgos secos que son introducidos cuando se utilizan los datos GTS en la calibración de los CMORPH. Esto sugiere que la calibración de los CMORPH con los datos de la red operativa requiere un estudio más cuidadoso y pormenorizado. Por otra parte en la Figura $11 \mathrm{~b}$, se puede apreciar el impacto de la calibración sobre las distribución de frecuencias en las estimaciones CMORPH. Como se puede apreciar en esta figura la calibración a pesar de mostrar un incremento en el sesgo seco de las estimaciones mejora en muchos aspectos la similitud de la distribución de lluvias estimadas con respecto a la distribución observada teniendo en cuenta el total de las observaciones SALLJEX+ANA.
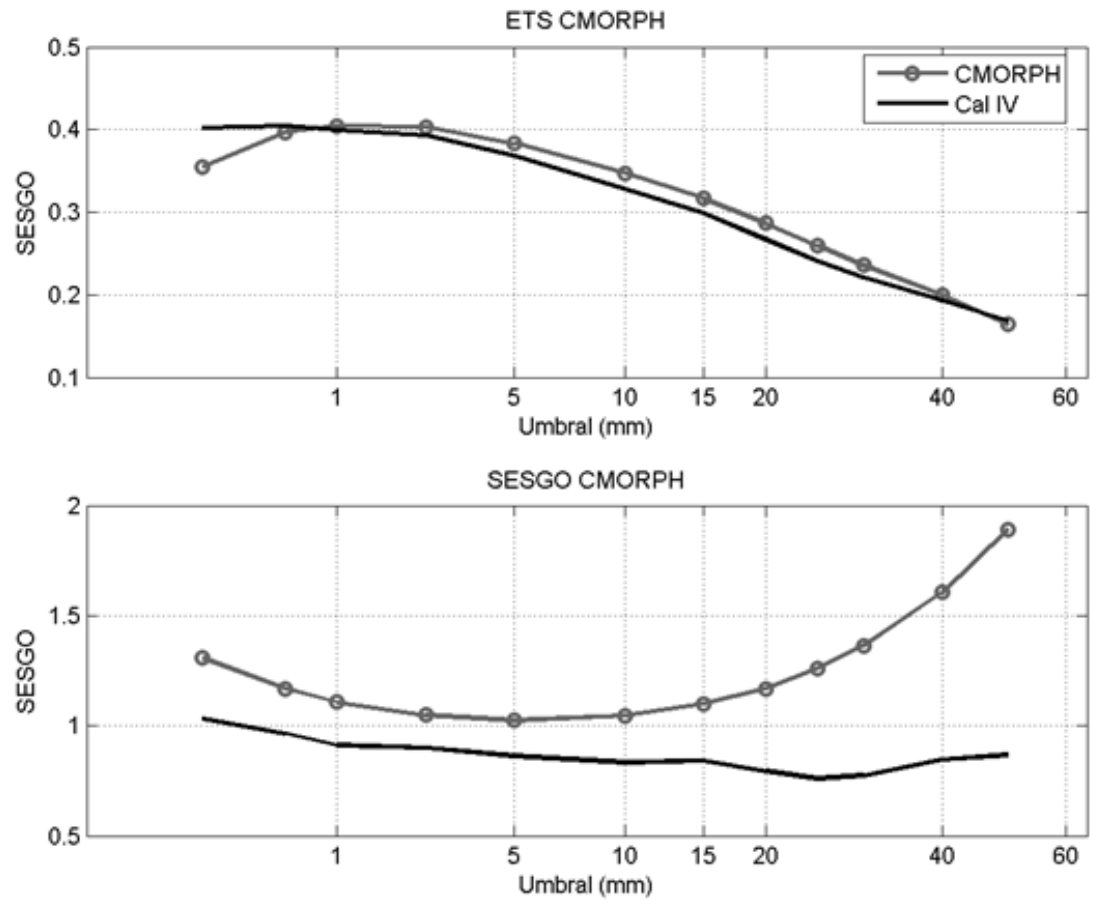

Figura 10 - Como en la Figura 9, pero para el experimento utilizando los datos del GTS. 
La mayor similitud de las estimaciones CMORPH calibradas a la distribución de precipitación observada se pone en evidencia también en la reducción de la diferencia cuadrática media entre las frecuencias observadas y las frecuencias estimadas.

El algoritmo propuesto a través de estos dos experimentos, demostró tener un desempeño satisfactorio aún cuando es aplicado a un período distinto del utilizado para el entrenamiento. Asimismo, la calibración puede ser aplicada aún cuando no exista información disponible para ese día en particular, dado que se basa exclusivamente en una estadística del comportamiento previo. Esto mismo sugiere que la calibración podría aplicarse a regiones aledañas o regiones con un escaso número de observaciones siempre y cuando las estadísticas de las diferencias entre CMORPH y las observaciones sean similares. De esta forma la calibración afecta en forma pareja y por igual a toda la estimación de precipitación sobre una región y no únicamente a aquellas regiones donde la densidad de datos es mayor.

También se realizaron experimentos para ver si la calibración obtenida durante la época estival, era aplicable a otros períodos del año. Para eso se aplicó la CAL IV obtenida durante la estación cálida 2002-2003 a un período comprendido entre octubre y diciembre de 2006. En este caso, los resultados obtenidos fueron poco satisfactorios (no se muestra la figura), el sesgo mostró que el producto calibrado induce una importante subestimación y los valores de ETS de la estimación calibrada mostraron ser muy inferiores a los no calibrados, sobre todo para los valores mayores de precipitación. No obstante al utilizar una calibración basada en datos correspondientes a dicho período, se obtuvieron resultados similares a los que se muestran en los

(A)

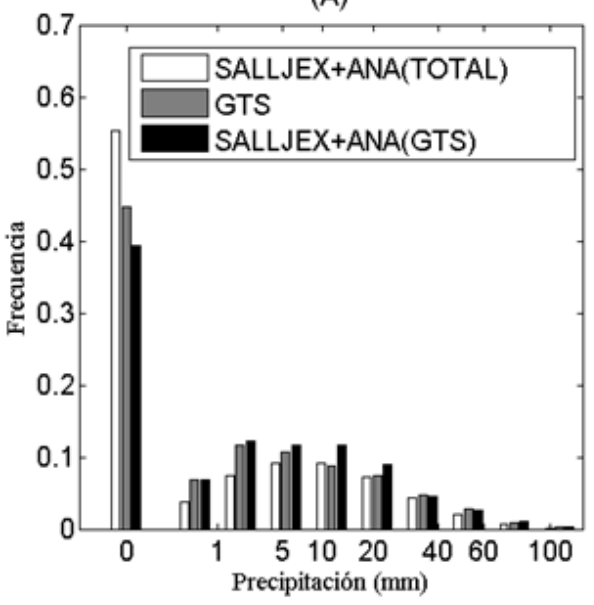

experimentos discutidos en este trabajo (reducción del sesgo sistemático con poco impacto en los valores de ETS). Este experimento marca la necesidad de incluir información sobre la estación del año en el proceso de calibración, lo cual está de acuerdo con lo encontrado por Joyce et al. (2004) y Ebert et al. (2007) entre otros en cuanto a la existencia de diferencias significativas e importantes en los valores de sesgo a lo largo del año en esta y otras estimaciones de precipitación. En particular, estos autores encontraron que para diferentes regiones analizadas el sesgo correspondientes a la época de invierno eran inferiores a los correspondientes a la época estival. Esto sugiere que una calibración basada en la remoción del sesgo no puede ser aplicada de igual manera durante la época estival o invernal. Como destacan Joyce et al. (2004) esta variación del sesgo a lo largo del año puede obedecer a las diferencias en el tipo de nubosidad que da origen a la precipitación en ambas estaciones, mientras que en el verano en la región analizada, predomina la precipitación de origen convectivo, durante el invierno, predomina la precipitación de origen estratiforme y el comportamiento del algoritmo parece ser fuertemente dependiente del origen de la lluvia.

Finalmente, para visualizar el impacto de la calibración en un evento particular se muestra el resultado de aplicar la CAL IV a un evento de mucha precipitación sobre el NE de Argentina entre los días 19 y 20 de diciembre de 2002 (Figura 12). Este período corresponde a la etapa final de un sistema convectivo sobre el sur de Brasil y norte de Uruguay y al desarrollo posterior de convección sobre el NE de Argentina. La precipitación asociada a la etapa final del sistema convectivo parece estar siendo subestimada, efecto que se acentúa al aplicar

gura 11 - Histograma de frecuencias relativas de precipitación para (a) Red SALLJEX+ANA sobre todos los puntos disponibles (barras blancas), red GTS (barras grises), red SALLJEX+ANA en las ubicaciones de las estaciones de la red GTS (barras negras) y (b) Red SALLJEX+ANA en todos los puntos disponibles (como en A) (barras blancas), estimaciones CMORPH sin calibrar sobre todos los puntos disponibles (barras grises), estimaciones CMORPH calibradas en base a los datos del GTS (barras negras). 

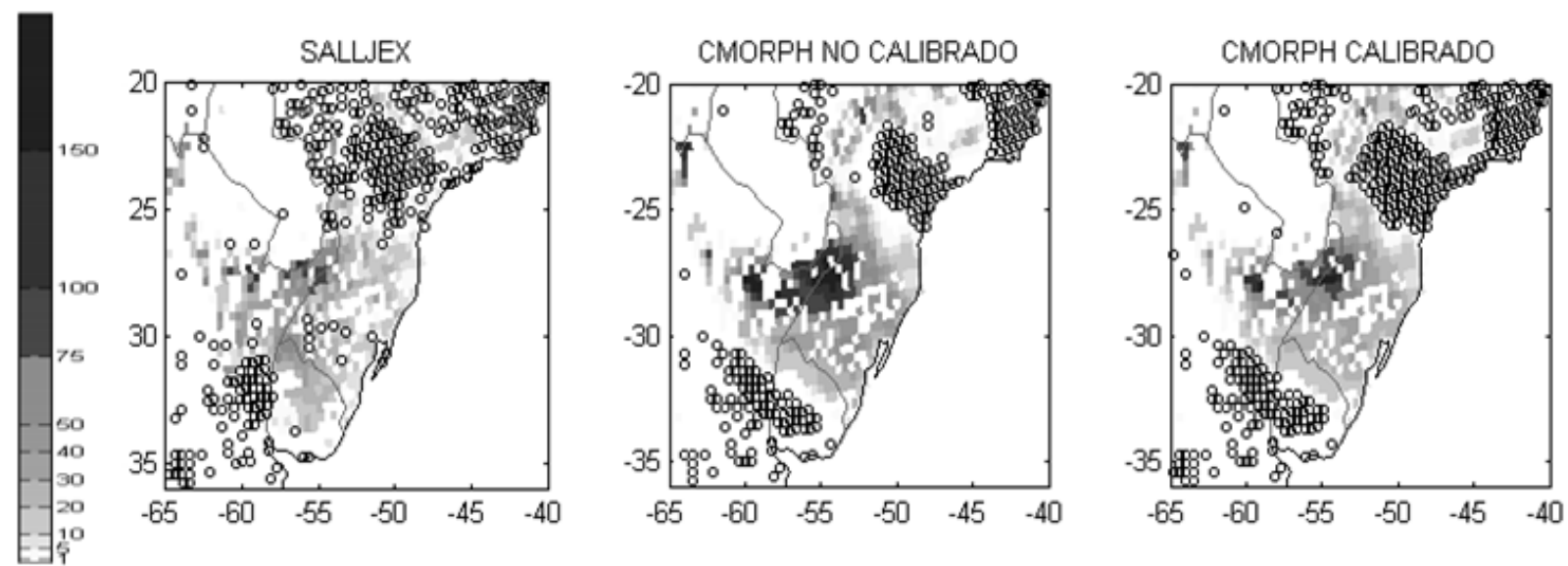

Figura 12 - Análisis de la distribución espacial de la precipitación entre las 12 UTC del 19 de diciembre y las 12 UTC del 20 de diciembre de 2002. (a) Precipitación medida en la red pluviométrica, (b) precipitación estimada por el CMORPH sin calibrar, (c) precipitación estimada por el CMORPH aplicando la calibración IV.

la calibración, mientras que la sobreestimación asociada a la región donde se desarrolla convección profunda en el NE de Argentina es reducida significativamente luego de la aplicación de la calibración, en mayor concordancia con la precipitación observada por la red pluviométrica. Si bien este es un caso particular, la precipitación asociada a la etapa final del sistema convectivo podría ser de naturaleza predominantemente estratiforme a diferencia de la actividad convectiva más vigorosa que se desarrollaba sobre el NE y el comportamiento observado podría corroborar para este caso particular la idea de que la estimación de la precipitación estratiforme muestra valores de sesgo inferiores con respecto a la convectiva.

\section{CONCLUSIONES}

Los experimentos analizados, muestran que la aplicación de una calibración permite reducir el error sistemático presente en las estimaciones CMORPH sobre la región del sur de Sudamérica. No obstante la componente no sistemática del error, o al menos la que no está vinculada con la intensidad de la lluvia es muy importante y depende de factores que escapan a lo analizado en el presente estudio. También se detectaron in homogeneidades en la distribución espacial de los errores sistemáticos de los CMORPH que requerirían de una calibración que se adapte a cada región en particular. El desarrollo de esta calibración es parte de un estudio en curso.

En este estudio solo se consideró la dependencia de los errores con el valor de precipitación estimado por el CMORPH, no obstante los errores podrían tener dependencias con otras variables como por ejemplo la concentración de aerosoles o el contenido de vapor integrado en la columna. En particular el segundo podría ser estimado a partir de análisis o pronósticos a corto plazo. Para incorporar otras variables en el marco de una calibración como la CAL IV, bastaría con realizar en forma independiente el cálculo de las curvas de calibración para diferentes rangos de las variables que queremos introducir en el modelo. Previamente se deben llevar a cabo estudios que muestren la sensibilidad de dichas curvas a las variables que se desea incorporar.

De todas las estrategias de calibración analizadas en el presente estudio, la aplicación de un algoritmo de reducción del sesgo basado en el trabajo de Hamill (1999) parece ser el que muestra mejores resultados y por construcción el que reduce más eficientemente el sesgo con respecto a la distribución de precipitación observada. Esto podría deberse a que esta calibración no se ve afectada por los errores en el posicionamiento de los sistemas que si afectan a las demás calibraciones con excepción de la calibración II.

La calibración mostró ser útil aún cuando era aplicada a un período distinto que el que había sido utilizado como entrenamiento, dentro de la misma estación del año. Esto marca además una diferencia con otras metodologías de incorporación de datos pluviométricos como la propuesta por Huffman et al. (1995) que depende de la disponibilidad de datos para cada día en particular para poder realizar la corrección. Según el algoritmo propuesto en este trabajo, la corrección se basa en los días previos y eventualmente puede aplicarse en días o regiones en donde los datos no estén disponibles o la densidad de estaciones sea muy escasa habiéndose demostrado previamente que el comportamiento de los errores sea lo suficientemente homogéneo. De esta manera, se pueden extender los resultados obtenidos en regiones donde existe una mayor densidad de 
datos hacia regiones cercanas, donde la densidad de datos es menor o donde en algún día en particular no se cuenta con información.

No obstante algunas limitaciones encontradas, los resultados obtenidos sugieren que la aplicación de calibraciones como la CAL IV, permitirían el aumento del valor de la información de los datos CMORPH para aplicaciones tales como la verificación de pronósticos, la calibración de pronósticos probabilísticos de precipitación y las aplicaciones hidrológicas tales como la determinación de la precipitación areal sobre una región o la asimilación de las estimaciones en modelos de suelo para el cálculo de la humedad del suelo. Por otra parte, la aplicación de este tipo de calibraciones utilizando la red de observaciones operativas no mostró resultados tan satisfactorios, por lo que el desarrollo de una calibración que pueda aplicarse en forma operativa a partir de una cantidad de datos menor es todavía una tarea pendiente. En este sentido es necesario profundizar en el estudio de si las diferencias en las funciones de distribución de probabilidad asociadas a diferentes redes obedecen solamente a las diferencias en el muestreo espacial o si existen otros factores.

Se ha comprobado que la calibración debe incluir información sobre la época del año, en concordancia con trabajos previos para diferentes regiones del mundo que muestran una importante variación de los errores sistemáticos de acuerdo con la estación del año (e.g. Joyce et al., 2004 y Ebert et al., 2007). Para la realización de una calibración válida para toda época del año, se pueden ensayar 2 alternativas, a saber: una calibración dinámica basada en las precipitaciones observadas en un período previo de aproximadamente un mes ó una calibración estática construida utilizando datos provenientes de varios años. Este tipo de estrategias han sido aplicadas exitosamente sobre la región para la calibración de pronósticos probabilísticos de precipitación (Ruiz et al., 2009). Al extender la calibración a otros períodos, surge la limitación de la menor cantidad de datos disponibles, ya que la red operativa de precipitación sobre la región es pobre. Cuando la calibración fue aplicada utilizando la red de estaciones operativas los resultados no fueron tan satisfactorios. Esto es tan solo uno de los motivos por los cuales es necesario incrementar la densidad y calidad de la información pluviométrica sobre la región.

\section{AGRADECIMIENTOS:}

El autor desea agradecer muy especialmente los valiosos comentarios de la Dra. Celeste Saulo que ayudaron a mejorar diversos aspectos de este trabajo y los comentarios de dos revisores anónimos los cuales contribuyeron a profundizar el análisis realizado sobre los errores sistemáticos de las estimaciones CMORPH sobre Sudamérica. Se desea agradecer además al Climate Prediction Center por permitir el acceso a las estimaciones de precipitación CMORPH y a los organizadores del experimento SALLJEX y a Dave Allured por proveer los datos pluviométricos. Los siguientes proyectos financiaron esta investigación: PICT 2003 07-14420, PICT $199907-$ 00000-06671, ambos de la Agencia Nacional de Promoción Científica, Tecnológica, X266 y X155 UBACYT de la Universidad de Buenos Aires y PIP 5417 del Consejo Nacional de Investigaciones Científicas y Técnicas.

\section{BIBLIOGRAFIA}

CAMPETELLA, M. C.; SAULO, A. C. Verificación Objetiva del Modelo LAHM/CIMA Sobre el Centro-Este de Argentina y Uruguay. Meteorologica, v. 28, p. 83-95, 2003.

CHOKNGAMWONG R.; CHIU L. S. Thailand Daily Rainfall and Comparison with TRMM Productos. J. Hydrometeor, v. 9, p. 256-266, 2008.

EBERT, E. E.; JANOWIAK, J. E.; KIDD, C. Comparison of Near-Real-Time Precipitation Estimates from Satellite Observations and Numerical Models. Bull. Amer. Meteor. Soc., v. 88, p. 47-64, 2007.

GEVAERD, R.; FREITAS, S. Estimativa Operacional da Umidade do Solo para Iniciação de Modelos de Previsão Numérica da Atmosfera, Parte I: Descrição da metodología e validação. RBMET, v. 21, p. 59-73, 2006.

HAMILL, T. Hipothesis Tests for Evaluating Numerical Precipitation Forecasts. Wea. Forecasting, v. 14, p.155-167, 1999.

HOSSAIN, F.; HUFFMAN, G. J. Investigating Error Metrics for Satellite Rainfall Data at Hydrologically Relevant Scales. J. Hydrometeor., v. 9, p. 563-575, 2008.

HUFFMAN, G. J.; ADLER, R. F.; BOLVIN, D. T.; GU, G.; NELKIN, E. J.; BOWMAN, K. P.; YANG, H.; STOKER, E. F.; WOLFF, D. B. The TRMM Multisatellite Precipitation Analysis (TMPA): Quasi-Global, Multiyear, Combined-Sensor Precipitation Estimates at Fine Scales. J. Hydrometeor., v. 8, p. 38-55, 2007.

HUFFMAN, G. J.; KRAJEWSKI, W. F.; MORRISSEY, M. L.; HUFFMAN, G. J.; ADLER, R. F. A Detailed Evaluation of GPCP $1^{\circ}$ Daily Rainfall Estimates over the Mississippi River Basin. J. App. Meteor., v. 44, p. 665-681, 2005.

JOYCE, R. J.; JANOWIAK J. E.; ARKIN, P. A.; XIE, P. CMORPH: A method that produces global precipitation estimates from passive microwave and infrared data at high spatial and temporal resolution. J. Hydrometeor., v. 5, p. 487-503, 2004.

KIDD, C. K.; KNIVETON, M. C.; TODD, M. C.; BELLERBY, T. J. Satellite rainfall estimation using combined passive 
microwave and infrared algorithms. J. Hydrometeor., v. 4, p. 1088-1104, 2003.

LIEBMANN, B.; ALLURED, D. Daily precipitation grids for South America. Bull. Amer. Meteor. Soc., v. 86, p. 15671570, 2005.

PENALBA, O.; VERA, C.; CERNE, B.; RUSTICUCCI, M.; SALIO, P.; FERREIRA, L.; LIEBMANN, B.; ALLURED, D.; DIAZ, A. : Daily Rainfall Data over Argentina and Uruguay during SALLJEX. CLIVAR Exchanges. v. 9, p. 29-31, 2004.

RUIZ, J. J.; SAULO, A. C.; KALNAY, E. Comparison of methods to generate probabilistic quantitative precipitation forecasts over South America, Wea. Forecasting., v. 24, p. 319-336, 2009

SAULO, A. C.; FERREIRA, L. Evaluation of quantitative precipitation forecasts over southern South America. Aust. Met. Mag. v. 52, p. 81-93, 2003.

SAURRAL, R.; BARROS, V.; LETTENMAIER, D. Land use impact on the Uruguay River discharge. Geophys. Res. Lett., v. 35, L12401, doi: 10.1029/ 2008GL033707
SCHAEFER, J. T. The Critical Success Index as an Indicator of Warning Skill. Wea. Forecasting, v. 5, p. 570-575, 1990.

SELUCHI, M. E.; CHOU, S. C. Ajuste del Esquema Convectivo de Betts-Miller en el Modelo Regional ETA/CPTEC. Meteorologica. v. 25, p. 45-56, 2000.

STENSRUD, D; YUSSOUF, N. Reliable Probabilistic Quantitative Precipitation Forecast from a Short-Range Ensemble Forecasting System. Wea. Forecasting, v. 22, p. 3-17, 2007.

SU, F.; YANG, H.; LETTENMAIER, D. Evaluation of TRMM Multi-satellite Precipitation Analysis (TMPA) and its utility in hydrologic prediction in La Plata Basin. J. Hydrometeor, v. 9, p. 622-640, 2008.

VERA, C.; BAEZ, J.; DOUGLAS, M.; EMMANUEL, C. B.; MARENGO, J.; MEITIN, J.; NICOLINI, M.; NOGUESPAEGLE, J.; PAEGLE, J.; PENALBA, O.; SALIO, P.; SAULO, C.; SILVA DIAS, M. A.; SILVA DIAS, P.; ZIPSER, E. The South American Low-Level Jet Experiment (SALLJEX). Bull. Amer. Meteor. Soc, v. 87, p. 63-77, 2006. 